\title{
Circulating leptin levels in juvenile idiopathic arthritis: a marker of nutritional status?
}

\author{
F Perfetto, R Tarquini, G Simonini, G Bindi, F Mancuso, S Guiducci, M Matucci-Cerinic, F Falcini
}

Background: Weight loss is common in juvenile idiopathic arthritis (JIA) and has been positively correlated with an increase in the production of proinflammatory cytokines. Objective: To assess if plasma leptin is a mediator of cytokine dependent decreased food intake during inflammatory diseases and if it is increased in JIA.

Methods: Leptin levels were determined in 31 patients with polyarticular disease and in 37 with oligoarticular disease; 32 healthy children served as controls.

Results: Patients had significantly reduced body mass index (BMI) compared with controls (17.3 (3) v 19.1 (3) kg/m²; $\mathrm{p}<0.005)$. Leptin was significantly lower in patients than controls (8.1 (4.8) v $10.7(7.3) \mathrm{ng} / \mathrm{ml} ; \mathrm{p}=0.036)$, but leptin/ $\mathrm{BMI}$ values were similar. Absolute (8.2 (4.8) v 8 (4.9); $\mathrm{p}>0.05)$ and normalised (0.45 (0.24) v $0.47(0.24)$; $\mathrm{p}>0.05$ ) leptin levels were not significantly different between patients with active and inactive disease and between patients with oligoarticular and polyarticular arthritis (7.8 (4.4) v 8.6 (5.3); $p>0.05$ and 0.45 (0.23) v 0.48 (0.26); $\mathrm{p}>0.05$, respectively).

Conclusions: Leptin production per unit of fat mass is similar in patients and controls. The hypothesis that high levels of proinflammatory cytokines that characterise JIA might induce an increase of adipocytes leptin production is not supported by the results. Leptin may be a marker of nutritional status of JIA.

A norexia and weight loss are consistent clinical manifestations during acute and chronic inflammatory processes, which are accompanied by decreased quality of life and survival. This change is generally attributed to the increased levels of circulating cytokines such as tumour necrosis factor $\alpha$, interleukins 1 (ILl) and 6 (IL6).

Leptin, the product of the ob/ob gene, is a secreted peptide derived from adipose tissue that is thought to be a lipostatic signal to brain centres coordinating metabolism. A positive correlation between body mass index (BMI) or body fat mass and circulating serum leptin concentrations has been (fig 1) reported. ${ }^{12}$ In animals, evidence has suggested that proinflammatory cytokines may up regulate adipocyte leptin production. $^{3}$ In humans, increased leptin levels have been reported in acute inflammatory and neoplastic conditions, suggesting that leptin may have a role in the acute phase response. ${ }^{45}$

Juvenile idiopathic arthritis (JIA) is an inflammatory joint disorder characterised by chronic synovitis and associated with extra-articular manifestations, such as fever, anaemia, lymphadenopathy, pericarditis, uveitis, and weight loss. IL1, IL6, and tumour necrosis factor $\alpha$ are the key cytokines that drive inflammation in JIA and may explain most of the articular and extra-articular manifestations. Because proinflammatory cytokines may up regulate leptin production, it has been suggested that cytokine related anorexia may, in part, be mediated through hyperleptinaemia. As far as we know, no data are available about the circulating leptin concentration in JIA. We aimed at studying the serum leptin levels in relation to disease activity and nutritional status in a cohort of patients with JIA.

\section{SUBJECTS AND METHODS}

Our study group included 68 children ( 25 male, 43 female, mean (SD) age 9.3 (4.4) years) who were affected by JIA and classified according to the Durban criteria. ${ }^{6}$ Thirty one had polyarticular, and 37 had oligoarticular onset disease; 6/37 patients with oligoarticular disease had extended oligoarthritis.

Patients were subdivided according to the clinical findings (fever, rash, joint inflammation) and erythrocyte sedimentation rate in a group of 26 patients with active disease and another of 42 patients with inactive disease. Polyarticular arthritis was defined as active in the presence of $(a)$ erythrocyte sedimentation rate $>25 \mathrm{~mm} / \mathrm{l}^{\mathrm{st}} \mathrm{h}$; $(b)$ swelling with reduced range of motion in more than five joints. Oligoarticular arthritis was defined as active in the presence of joint swelling with reduced range of motion in one to four joints.

Twenty five patients with polyarthritis and six with extended oligoarthritis were receiving methotrexate (MTX) treatment. No patients were receiving or had received oral or intra-articular corticosteroids before the start of the study. BMI was calculated as weight/height ${ }^{2}$. Thirty two healthy children (11 male, 21 female; mean (SD) age 9.7 (4.9) years) attending our outpatient clinic for musculoskeletal pain and without signs of inflammation served as controls. Approval was obtained from the ethical committee of Meyer Hospital and parents or guardians gave informed consent.

Venous blood samples were collected in a chilled tube at $0800 \mathrm{am}$ after overnight fasting. The plasma leptin concentration $(\mathrm{ng} / \mathrm{ml})$ was determined by radioimmunoassay (kit provided by DRG Instruments GmbH, Postfach, Marburg, Germany). Results were expressed as mean (SD). The data were examined by analysis of variance and single and multiple regression analysis. Statistical significance was accepted at $5 \%$ probability levels.

\section{RESULTS}

Patients and controls were of similar age, sex, and height, but they differed in weight (30.6 (14) $v 36.9$ (14) kg; $\mathrm{p}<0.04)$ and BMI (17.3(3) v 19.1 (3) $\mathrm{kg} / \mathrm{m}^{2} ; \mathrm{p}<0.005$ ) (table 1 ). Plasma leptin levels were significantly lower in patients than in controls (8.1 (4.8) $v 10.7$ (7.3) ng/ml; $\mathrm{p}=0.036)$, but this difference was no longer significant when leptin concentration was corrected according to the BMI of the two groups

Abbreviations: BMI, body mass index; IL, interleukin; JIA, juvenile idiopathic arthritis; MTX, methotrexate; RA, rheumatoid arthritis 


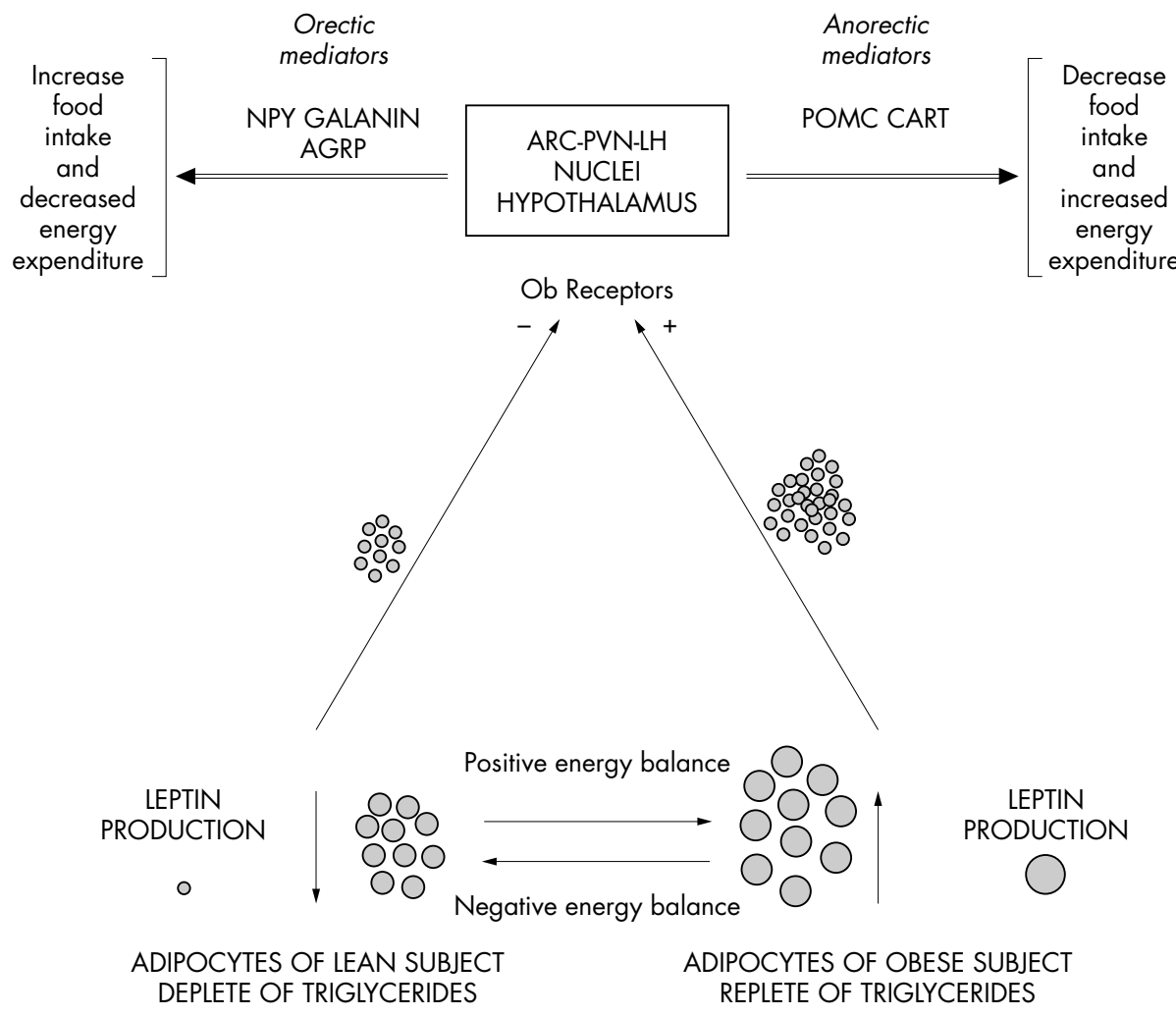

Figure 1 Proposed mechanism of action of leptin. The adipocytes actively modulate energy balance through secretion of leptin. Leptin secretion increases when fat cells are replete with triglycerides. Leptin travels through the circulation, crosses the blood-brain barrier, acts on leptin receptors (Ob receptors) located on several neuronal subtypes within the hypothalamus and elicits neuroendocrine and autonomic nervous system responses, which decrease food intake and increase energy expenditure (right of fig). The depletion of adipocytes by triglycerides causes a proportionally decrease in leptin secretion, which at hypothalamic levels increases food intake and decreases energy expenditure (left of fig). ARC: arcuate nucleus; PVN, paraventicular nucleus; LH: lateral hypothalamus; Orectic mediators: NPY, neuropeptide Y; AGRP, agouti related peptide; GALANIN. Anorectic mediators: POMC, propiomelanocortin; CART, cocaine and amphetamine regulated transcript.

(relative leptin concentrations expressed as leptin/BMI: 0.46 (0.24) $\left.v 0.54(0.29)(\mathrm{ng} / \mathrm{ml}) /\left(\mathrm{kg} / \mathrm{m}^{2}\right) ; \mathrm{p}>0: 05\right)$ (fig 2). We found similar absolute and relative leptin concentrations in patients with active and inactive disease (8.2 (4.8) $v 8$ (4.9) and $0.45(0.24) \vee 0.47(0.24)$, respectively; $p>0.05$ for both) and in children with oligoarticular and polyarticular disease $\left.\begin{array}{llllllllll}7.8 & (4.4) & v & 8.6 & (5.3)\end{array}\right)$ and $0.45 \quad(0.23) \quad v \quad 0.48$ (0.26), respectively; $\mathrm{p}$, NS, table 2 ).

Patients treated with MTX showed similar absolute (8.7 (5.4) $v 7.7$ (4.5); $\mathrm{p}>0.05$ ) and relative leptin concentrations $(0.49(0.26) v 0.44(0.23) \mathrm{p}>0.05)$ to those of patients not treated with MTX. Girls showed significantly higher leptin concentrations than boys (in patients: 9.1 (5) $v 6.6$ (4.1) ng/ $\mathrm{ml}, \mathrm{p}<0.05$; in controls: 12.7 (7.9) $v 7$ (4.1) ng/ml, p<0.03). These differences were amplified when leptin values were adjusted for BMI (in patients: $0.52(0.25) \vee 0.36(0.19)$

Table 1 Clinical and anthropometric characteristic of 68 children with juvenile idiopathic arthritis and 32 healthy controls

\begin{tabular}{llll}
\hline Characteristics & Controls & Patients & p Values \\
\hline No of subjects & 32 & 68 & - \\
Sex (female/male) & $21 / 11$ & $43 / 25$ & - \\
Age (years) & $9.7(4.9)$ & $9.3(4.4)$ & $\mathrm{NS}$ \\
Height $(\mathrm{m})$ & $1.35(0.22)$ & $1.29(0.23)$ & $\mathrm{NS}$ \\
Weight (kg) & $36.9(14)$ & $30.6(14)$ & $<0.04$ \\
Body mass index & $19.1(3)$ & $17.3(3)$ & $<0.005$ \\
$\left(\mathrm{~kg} / \mathrm{m}^{2}\right)$ & & \\
\hline \multirow{2}{*}{ Results are shown as mean (SD). }
\end{tabular}

$(\mathrm{ng} / \mathrm{ml}) /\left(\mathrm{kg} / \mathrm{m}^{2}\right), \mathrm{p}<0.008$; in controls: $0.63(0.30) \vee 0.36$ (0.17) $\left.(\mathrm{ng} / \mathrm{ml}) /\left(\mathrm{kg} / \mathrm{m}^{2}\right), \mathrm{p}<0.009\right)$.

Leptin concentration correlated positively with BMI in patients and controls $(r=0.552$ and $r=0.744$, respectively; $\mathrm{p}<0.0001$ for both). In patients, leptin correlated positively also with age $(r=0.407, \mathrm{p}<0.0005)$. Multiple regression analysis indicated that BMI together with sex $(p<0.001$ for both) were the best predictors of circulating leptin in patients, accounting for $66 \%$ of its variance. Some other measures (disease activity and MTX treatment) could be included in the model without a significant change in its strength. In controls, BMI, sex, and age were the best predictors of circulating leptin, accounting for $81 \%$ of its variance.

\section{DISCUSSION}

This study indicates that leptin production per unit of fat mass is similar in patients with JIA and controls and that the lower BMI found in patients with JIA could account for their low circulating leptin concentrations. Moreover, multiple regression analysis showed that neither the activity of the disease nor the type of onset of disease correlated with circulating leptin concentrations. In agreement with published reports, BMI and sex were the most important independent predictors of plasma leptin levels in patients and controls. Thus, the hypothesis that leptin might be the mediator of anorexia and weight loss in JIA, is not supported by our results. Few studies have examined the correlation between leptin and rheumatic disease and, to date, this is the first report on leptin in JIA. 

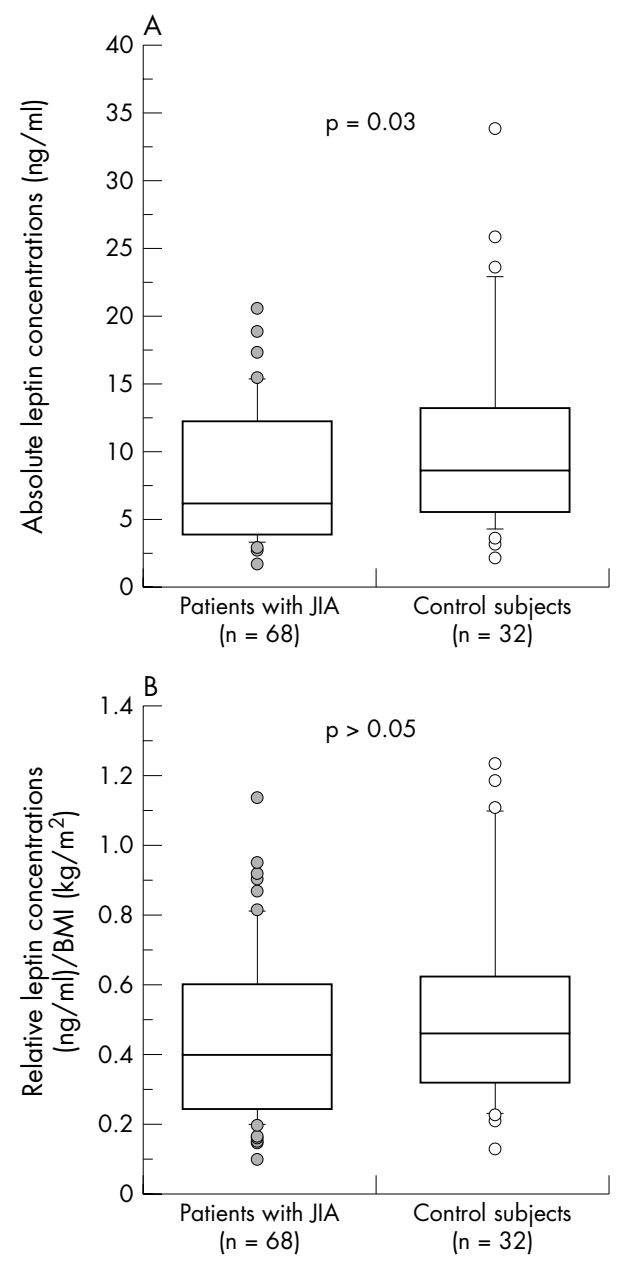

Figure 2 (A) Significant differences of absolute serum leptin concentrations $(\mathrm{ng} / \mathrm{ml}$ ) between patients with JIA and healthy controls. (B) This difference disappeared when leptin levels were corrected for BMI values of subjects studied and expressed as a leptin/BMI ratio.

In patients with rheumatoid arthritis (RA), leptin concentrations were found to be similar to those of healthy controls $^{78}$ and were unrelated to disease activity. Bokarewa et al reported that leptin plasma levels were significantly higher than leptin concentrations in RA matched synovial fluid samples." Plasma and synovial fluid leptin were significantly correlated, but had no relationship with age, sex, and disease duration. Furthermore, patients with nonerosive arthritis had lower leptin concentrations in synovial fluid, suggesting an in situ consumption of this peptide. They concluded that intra-articular leptin might exert a protective effect against the destructive course of RA.

Although leptin levels were reported to be higher in patients with systemic lupus erythematosus than in controls, ${ }^{10}$ leptin did not correlate with disease activity index, whereas a positive relationship was found in patients with Behçet's disease. ${ }^{11}$ As suggested by Palmer and Gabay in a recent review of the role of leptin in rheumatic diseases, ${ }^{12}$ these data indicate that leptin cannot be used to assess disease activity in RA and systemic lupus erythematosus.

Our data are consistent with reports that failed to show increased leptin concentrations in chronic human cytokine mediated disorders such as HIV infection, inflammatory bowel disease, and neoplastic cachexia. In all these inflammatory diseases, plasma leptin concentrations correlated strongly with BMI.
Table 2 Clinical characteristics and serum leptin levels of 68 children with oligoarticular and polyarticular juvenile idiopathic arthritis (JIA)

\begin{tabular}{|c|c|c|c|}
\hline Characteristics & $\begin{array}{l}\text { Oligoarticular } \\
\text { JIA }\end{array}$ & $\begin{array}{l}\text { Polyarticular } \\
\text { JIA }\end{array}$ & p Values \\
\hline No of subjects & 37 & 31 & - \\
\hline Sex (female/male) & $27 / 10$ & $17 / 14$ & - \\
\hline Age (years) & $9.1(4.2)$ & $9.6(4.7)$ & NS \\
\hline Height $(\mathrm{m})$ & $1.30(0.22)$ & $1.27(0.24)$ & NS \\
\hline Weight (kg) & $30.8(14)$ & $30.4(15.9)$ & NS \\
\hline $\begin{array}{l}\text { Body mass index } \\
\left(\mathrm{kg} / \mathrm{m}^{2}\right)\end{array}$ & $17.2(2.6)$ & $17.4(3.4)$ & NS \\
\hline Leptin (ng/ml) & $7.8(4.4)$ & $8.6(5.3)$ & NS \\
\hline $\begin{array}{l}\text { Leptin /BMI ((ng/ml)/ } \\
\left.\left(\mathrm{kg} / \mathrm{m}^{2}\right)\right)\end{array}$ & $0.45(0.23)$ & $0.48(0.26)$ & NS \\
\hline
\end{tabular}

The finding that in our patients low leptin concentrations were not reflected by an increase in body weight may suggest that the hypothalamus is insensitive to low circulating leptin concentrations and that the normal homoeostatic mechanism that protects body weight against losses has been overridden. A similar mechanism has been proposed by Inui for the pathophysiology of the cancer anoroxia-cachexia syndrome. ${ }^{13}$ The mechanism of this hypothalamic insensitivity to low circulating leptin levels is not clear, but there is evidence that proinflammatory cytokines may mime the hypothalamic effect of excessive negative feedback signalling from leptin, leading to the prevention of the normal compensatory mechanisms in the face of both decreased food intake and decreased body weight. This appears to be a likely explanation because the leptin receptor is homologous to the gp130 signal transducing molecule associated with the IL6-type cytokine receptor and shares the same post-receptor signalling pathway via activation of the signal transducers and activators of the transcription family, mainly through stat 3 protein activation. ${ }^{14}$ Therefore, as stat 3 protein is essential for leptin induced anorexia, the cytokines that share with leptin the same post-receptor signalling pathway could likewise induce anorexia and unopposed weight loss.

In agreement with published reports, ${ }^{15}$ girls had higher leptin concentrations than boys despite having similar BMIs. The reason for this difference between the sexes is unclear, but several studies ${ }^{15}$ report that girls' fat cells produce more leptin than those of boys with a similar body mass.

In conclusion, our results suggest that weight loss in JIA is not due to an increase in leptin levels. In JIA, circulating leptin levels that are merely a function of the amount of body fat do not reflect disease activity but may be a biochemical marker of patient nutritional status. Further longitudinal studies elucidating the interaction between leptin, proinflammatory cytokines, and acute phase proteins might provide important insights into the pathways involved in energy balance regulation and anorexia in JIA.

\section{Authors' affiliations}

F Perfetto, R Tarquini, F Mancuso, S Guiducci, M Matucci-Cerinic, Department of Internal Medicine, Rheumatology Unit, University of Florence, Italy

G Simonini, G Bindi, F Falcini, Department of Paediatrics, Rheumatology Unit, University of Florence, Italy

Correspondence to: Dr F Perfetto, Department of Medicine, Section of Rheumatology, University of Florence, Viale Pieraccini 19; 50139 Florence, Italy; perfetto@unifi.it

Accepted 16 May 2004 


\section{REFERENCES}

1 Friedman JM, Halaas $\mathrm{JL}$. Leptin and the regulation of body weight in mammals. Nature 1998:395:763-70.

2 Kalra SP, Dube MG, Pu S, Xu B, Horvath TL, Kalra PS. Interacting appetiteregulating pathways in the hypothalamic regulation of body weight. Endocrinol Rev 1999;20:68-100.

3 Sarraf P, Frederich RC, Turner E. M, Ma G, Jaskowiak NT, Rivet DJIII, et al. Multiple cytokines and acute inflammation raise mouse leptin levels: potentia role in inflammatory anorexia. J Exp Med 1997:185:171-5.

4 Bornstein SR, Licinio J, Tauchnitz R, Engelmann L, Negrao AB, Gold P, et al. Plasma leptin levels are increased in survivors of acute sepsis: associated loss of diurnal rhythm in cortisol and leptin secretion. J Clin Endocrinol Metab 1998;83:280-3.

5 Moses AGW, Dowidar N, Holloway B, Waddel I, Fearon KGH, Ross JA. Leptin and its relation to weight loss, ob gene expression and the acute-phase response in surgical patients. Br J Surg $2001 ; 88: 588-93$.

6 Petty RE, Southwood TR, Baum J, Bhettay E, Glass DN, Manners P, et al. Revision of the proposed classification criteria for juvenile idiopathic arthritis Durban 1997. J Rheumatol 1998;25:1991-4.

7 Anders HJ, Rihl M, Heufelder A, Loch O, Schattenkirchener M. Leptin serum levels are not correlated with disease activity in patients with rheumatoid arthritis. Metabolism 1999;48:745-8.
8 Nishiya K, Nishiyama M, Chang A, Shinto A, Hashimoto K. Serum leptin levels in patients with rheumatoid arthritis are correlated with body mass index. Rinsho Byari 2002;50:524-7.

9 Bokarewa M, Bokarew D, Hultren O, Tarkowsy A. Leptin consuption in the inflamed joints of patients with rheumatoid arthritis. Ann Rheum Dis 2003;62:952-6.

10 Garcia-Gonzalez A, Gonzalez-Lopez L, Valera-Gonzalez IC, CardonaMunoz EG, Salazar-Paramo M, Gonzalez-Ortiz M, et al. Serum leptin levels in women with systemic lupus erythematosus. Rheumatol Int 2002;22:138-41.

11 Evereklioglu C, Inaloz HS, Kirtak N, Daganay S Bulbul M, Ozerol E, et al. Serum leptin concentration is increased in patients with Behçet's syndrom and is correlated with disease activity. Br J Dermatol 2002;147:331-6.

12 Palmer G, Gabay C. A role for leptin in rheumatic diseases? Ann Rheum Dis 2003;62:913-15.

13 Invi A. Cancer anorexia-cachexia syndrome; are neuropeptides the key? Cancer Res 1999:59:4493-501.

14 Vaisse C, Halaas JL, Horvath CM, Darnell JE Jr, Stoffel M, Friedman JM. Leptin activation of stat 3 in the hypothalamus of wild-type and ob/ob mice but not $\mathrm{db} / \mathrm{db}$ mice. Nat Genet 1996;13:95-8.

15 Saad M, Damani M, Gingerich RL, Riad-Gabriel MG, Khan A, Boyadjian R, et al. Sexual dimorphism in plasma leptin concentration. J Clin Endocrinol Metab 1997;82:679-84. 